\title{
Multi-Vehicle Path Planning in Dynamically Changing Environments
}

\author{
Ali Ahmadzadeh ${ }^{1}$, Nader Motee $^{2}$, Ali Jadbabaie ${ }^{1}$ and George Pappas ${ }^{1}$
}

\begin{abstract}
In this paper, we propose a path planning method for nonholonomic multi-vehicle system in presence of moving obstacles. The objective is to find multiple fixed length paths for multiple vehicles with the following properties: (i) bounded curvature (ii) obstacle avoidant (iii) collision free. Our approach is based on polygonal approximation of a continuous curve. Using this idea, we formulate an arbitrarily fine relaxation of the path planning problem as a nonconvex feasibility optimization problem. Then, we propound a nonsmooth dynamical systems approach to find feasible solutions of this optimization problem. It is shown that the trajectories of the nonsmooth dynamical system always converge to some equilibria that correspond to the set of feasible solutions of the relaxed problem. The proposed framework can handle more complex mission scenarios for multi-vehicle systems such as rendezvous and area coverage.
\end{abstract}

\section{INTRODUCTION}

The problem of path planning for a vehicle in a dynamically changing environment has been an active research area in robotics and control communities [1], [2]. The major trends have been focused on holonomic and non-holonomic kinematic path planning problems. Perhaps Dubins' seminal work [3] is one of the first ones in this area that characterizes shortest bounded-curvature paths for a vehicle in absence of obstacles. It is well-known that finding a shortest boundedcurvature path amidst polygonal obstacles in the plane is NP-hard [4]. Also, researchers have shown that the general feasibility algorithm is exponential in time and space [5]. These results imply that struggling to find an efficient and exact algorithm to solve curvature-constrained path planning problem is hopeless. This partially resulted in developing various approximate methods to solve the path planning problem [6]- [7]. Nevertheless, the existing algorithms are incomplete in the sense that they may not provide a solution even if one exists.

Among different approaches to the path planning problem, the navigation function method is the closest one to our methodology [8]. In this method, the vehicles are steered by some artificially generated forces, defined as the negative gradient of a navigation function. The navigation function is defined so that it can generate attractive forces toward the goal and repulsive forces in the neighborhood of an obstacle.

\footnotetext{
1 The authors are with the Department of Electrical and Systems Engineering and GRASP Laboratory, University of Pennsylvania, 200 South 33rd Street, Philadelphia, PA 19104 USA ( $\{$ aliahmad, jadbabai, pappasg\}@ seas. upenn.edu).

2 The author is with the Control and Dynamical Systems, California Institute of Technology, 1200 E. California Blvd, Pasadena, CA 91125 USA (motee@cds.caltech.edu).
}

The main disadvantage of the navigation function methods is that they can not handle nonholonomic constraints such as bounded-curvature constraint.

In this paper, our goal is to propose a near-optimal and scalable method for solving the bounded-curvature path planning problem in presence of moving obstacles. We assume that each obstacle can be represented as union of nonoverlapping disks and their motion trajectories are known. First, we consider the path planning problem for a single vehicle. Then we extend our results to handle multi-vehicle path planning problems. Our approach is based on polygonal approximation of a continuous curve in the plane. A path connecting the initial and final positions of a vehicle can be approximated by finitely many waypoints. This approximation can be arbitrarily improved by increasing the number of waypoints. In this setting, we can relax the boundedcurvature and collision-free constraints by verifying the constraints only at these waypoints. This relaxation results in a finite-dimensional formulation of the path planning problem as a nonconvex feasibility optimization problem. Every feasible solution to the relaxed problem is an approximate bounded-curvature and collision-free path for the vehicle.

Furthermore, we propose a nonsmooth dynamical systems approach to find feasible solutions of the optimization problem. In this method, each waypoint is treated as a moving particle in the plane. We define interaction forces between the particles such that: (i) the set of equilibria of the system contains all feasible solutions of the optimization problem, and (ii) the corresponding multi-particle system is asymptotically stable. In an equilibrium point the net force on each particle is equal to zero. It is shown that by applying some specific type of nonsmooth interaction forces, the net force on each particle is equal to zero if and only if these particles are representing a feasible path. In other words, for every initial condition the trajectory of the system always converges to a feasible path for the vehicle. Since we are using discontinuous dynamical system, we need nonsmooth analysis and stability of nonsmooth systems to analyze the dynamical system with discontinuous right-hand sides. When studying a discontinuous vector field the classical notion of solution for dynamical system is too restrictive and may not even exist. There are several solution notions for discontinuous systems such as Caratheodory solutions, Krasovskii solutions and Filipov notion of solutions [13]. In this paper, we employ the notion of Filipov's solutions. Filipov in his seminal contribution [10] developed a solution concept for differential equations whose right-hand sides 
were only required to be Lebesgue measurable in the state and time variables. For our analysis, we will apply Shevitz and Paden's results [14] on nonsmooth Lyapunov stability theory and LaSalles invariance principle for a class of nonsmooth Lipschitz continuous Lyapunov functions.

This paper is organized as follows. In Section II, we formulate the path planning problem for a single vehicle as a feasibility optimization problem. A dynamical system approach to the path planning problem is discussed in Section III. In Section IV, it is shown that by using discontinuous interaction forces we can always guarantee the convergence of the trajectories of the system to feasible paths. The singlevehicle path planning in presence of moving obstacles is presented in Section V. In Section VI, we show that our methodology can be directly applied to the multi-vehicle path planning problem in presence of moving obstacles.

\section{Problem Formulation}

The goal of this paper is to find a fixed-length bounded curvature trajectory for a vehicle with given initial and final configurations in a dynamically changing environment. We assume that the dubins vehicle is traveling with a constant speed $V$. Suppose that there are $M$ moving obstacle with known motion patterns in the environments. At any time instant $t$, each obstacle is assumed to be represented by a $\operatorname{disk} \mathbf{D}\left(c_{j}(t), r_{j}(t)\right)=\left\{x \mid\left\|x-c_{j}(t)\right\| \leq r_{j}(t)\right\}$. We also assume that these disks are not overlapping for all time.

Path Planning with Moving Obstacles: Let $\kappa_{\max }>0$ be the maximum allowable curvature and $P, Q \in \mathbb{R}^{2}$ the initial and final points. Then the problem consists of finding a curve $\gamma:[0, T] \rightarrow \mathbb{R}^{2}$ (parameterized by time where $T>0$ is a fixed number) such that

$$
\begin{array}{ll}
\text { (i) } & \gamma(0)=P \text { and } \gamma(T)=Q . \\
\text { (ii) } & \kappa(t) \leq \kappa_{\max } \text { for all } t \in[0, T] . \\
\text { (iii) } & \gamma(t) \cap \mathbf{D}\left(c_{j}(t), r_{j}(t)\right)=\emptyset \text { holds for all } t \in[0, T] \\
& \text { and } j=1, \ldots, M .
\end{array}
$$

Note that $\kappa(t)$ is the curve curvature at time $t$. One can see that $\gamma$ is a fixed length curve of length $l=V T$. We refer to the second condition as the obstacle avoidance constraint. The third condition guarantees a bounded curvature curve.

In the sequel, we will tackle this problem in several steps and propose an arbitrarily fine approximation of the optimal solution. In Section II-A, we review polygonal approximation of a continuous curve with equidistant waypoints in $\mathbb{R}^{2}$. In Section II-B and II-C, we show that conditions (ii) and (iii) can be relaxed by verifying the constraints only at waypoints. In Section II-D, we will see that the path planning problem reduces to a feasibility optimization problem.

\section{A. Polygonal Curve Approximation}

Our approach is based on discrete approximation of a continuous curve using finite number of vertices. Consider a polygonal curve $\gamma_{p}=\overline{p_{0} p_{1} \ldots p_{n}}$ represented by its ordered vertices $p_{0}, p_{1}, \ldots, p_{n} \in \mathbb{R}^{2}$ where $p_{0}=P, p_{n}=Q$ and $\overline{p_{i} p_{i+1}}$ is the line segment connecting $p_{i}$ to $p_{i+1}$. Under some mild assumptions, for a given error bound $\epsilon>0$, one can always find points $\left\{p_{0}, p_{1}, \ldots, p_{n}\right\}$, for a large number $n>0$, such that

$$
\left|L\left(\gamma_{p}\right)-l\right|<\epsilon,
$$

where

$$
L\left(\gamma_{p}\right)=\sum_{i=1}^{n}\left\|p_{i}-p_{i-1}\right\| .
$$

Without loss of generality, we may assume that all points $p_{i}$ are equidistant. Therefore, it follows that

$$
d=\left\|p_{i}-p_{i-1}\right\| \simeq \frac{l}{n} .
$$

for all $i=1, \ldots, n$.

\section{B. Discrete Curvature}

If we assume that $d \ll \frac{1}{\kappa_{\max }}$, then we can use $C_{i}$ the circle passing through the points $\left(p_{i-1}, p_{i}, p_{i+1}\right)$ (if not all of these three points lie on a line), as an approximation to the osculating circle to the curve at point $p_{i}$ to calculate the curve curvature at that point. As both $p_{i-1}$ and $p_{i+1}$ move toward $p_{i}$, circle $C_{i}$ approaches a limiting circle with radius $r_{i}$ which is the same as the osculating circle at point $p_{i}$. More importantly, $\frac{1}{r_{i}}$ is the curvature at $p_{i}$. Therefore, we can employ circle $C_{i}$ to calculate an approximation of the curvature at point $p_{i}$. Let $A$ denotes the area of the triangle formed by nodes $\left(p_{i-1}, p_{i}, p_{i+1}\right)$ and $d_{i j}=\left\|p_{i}-p_{j}\right\|$. The discrete curvature $\kappa_{i}$ at point $p_{i}$ is defined by

$$
\kappa_{i}=\frac{1}{R_{i}}=\frac{4 A}{d_{(i-1) i} d_{i(i+1)} d_{(i-1)(i+1)}}
$$

By applying assumption (2) and the fact that the area of the triangle is $A=\sqrt{s(s-a)(s-b)(s-c)}$ where $s=\frac{a+b+c}{2}$, we have

$$
\kappa_{i}=\frac{2 \sqrt{d^{2}-\frac{d_{(i-1)(i+1)}^{2}}{4}}}{d^{2}} .
$$

By imposing the following constraint on discrete curvature

$$
\kappa_{i} \leq \kappa_{\max }
$$

it follows that

$$
\left\|p_{i-1}-p_{i+1}\right\|=d_{(i-1)(i+1)} \geq \frac{l}{n} \sqrt{4-\frac{\kappa_{\max }^{2} l^{2}}{n^{2}}}=\eta
$$

where $i=1, \ldots, n-1$.

\section{Moving Obstacles}

Our goal is to find a path for the Dubins vehicle in presence of moving obstacles with known motion patterns. Suppose that $t_{i}$ is the time instant at which the vehicle is at waypoint $p_{i}$. Therefore, the obstacle avoidance condition (iii) can be written as follow

$$
\left\|p_{i}-c_{j}\left(t_{i}\right)\right\| \geq r_{j}\left(t_{i}\right)
$$

for all $i=0, \ldots, n$ and $j=1, \ldots, M$. We assume that

$$
\left\|P-c_{j}(0)\right\| \geq r_{j}(0) \text { and }\left\|Q-c_{j}(T)\right\| \geq r_{j}(T)
$$

for all $j=1, \ldots, M$. 


\section{Relaxed Path Planning Problem}

A relaxation of the path planning problem can be posed as the following problem.

Relaxed Path Planning Problem as a Feasibility Problem: There exists a polygonal curve $\gamma_{p}=\overline{p_{0} p_{1} \ldots p_{n}}$ that satisfies conditions (i)-(iii) if and only if the following optimization problem is feasible

$$
\min _{\left\{p_{1}, \ldots, p_{n-1}\right\} \in \mathbb{R}^{2}} 0
$$

subject to: $p_{0}=P$ and $p_{n}=Q$,

$$
\begin{aligned}
& \left\|p_{i}-p_{i-1}\right\|=d, \quad i=1, \ldots, n \\
& \left\|p_{i-1}-p_{i+1}\right\| \geq \eta, \quad i=1, \ldots, n-1 \\
& \left\|p_{i}-c_{j}\left(t_{i}\right)\right\| \geq r_{j}\left(t_{i}\right), \quad i=1, \ldots, n-1 \\
& \quad j=1, \ldots, M .
\end{aligned}
$$

where $\eta$ is defined in (5). The optimization problem (7) is a nonconvex problem. In the following section, we we propose a multi-particle dynamical system approach to solve the feasibility problem (7). First, we consider the path planning problem without obstacles.

\section{Path Planning Using Stable Multi-Particle SYSTEMS}

In this section, we propose a method to find a feasible solution of problem (7) for a single vehicle in the absence of obstacles in the environment. Consider the waypoints $p_{0}, \ldots, p_{n} \in \mathbb{R}^{2}$. These points can be viewed as point mass particles moving on the plane with some initial random positions. Let $m_{i}$ be the mass of particle $i$ with position $p_{i}$. A force vector $\mathbf{F}_{i}$ can be associated to point mass particle $p_{i}$. Therefore, we have

$$
m_{i} \ddot{p}_{i}=\mathbf{F}_{i}
$$

where $i=0, \ldots, n$. Let $p=\left[p_{0}^{T}, p_{1}^{T}, \ldots, p_{n}^{T}\right]^{T}$ denote the state of the overall system. One can impose the following constraints

$$
p_{0}=P \quad \text { and } \quad p_{n}=Q
$$

on particles 0 and $n$ by assuming that $m_{0}, m_{n}>M$ for any large number $M>0$. In other words, two heavy masses are concentrated at points $P$ and $Q$ and that their positions are fixed.

Our goal is to design force vectors $\mathbf{F}_{i}$ for each particle such that the set of stable equilibria of the dynamical systems (8) is equal to the set of all feasible solutions of the optimization problem (7).

Definition 1: We refer to a real-valued function $f_{i j}$ as elasticity function if it satisfies the following conditions:

(i) $\quad f_{i j}=f_{j i}$ for all $i$ and $j$.

(ii) Functions $f_{i j}$ are nondecreasing.

(iii) The vector $\left(p_{0}, \ldots, p_{n}\right)$ is a feasible solution of problem (7) if and only if $f_{i j}\left(\left\|p_{i}-p_{j}\right\|\right)=0$ for all $i, j=0, \ldots, n$.
Throughout the paper, we will also refer to the elasticity functions as spring-like forces.

Theorem 1: All feasible solutions of problem (7) are stable equilibria of the multi-particle system (8) with

$$
\mathbf{F}_{i}=\sum_{\substack{j=0 \\ j \neq i}}^{n} f_{i j}\left(\left\|p_{i}-p_{j}\right\|\right) \mathbf{e}_{i j}-v \dot{p}_{i}
$$

where $f_{i j}$ 's are continuous spring forces, $\mathbf{e}_{i j}=\frac{p_{j}-p_{i}}{\left\|p_{i}-p_{j}\right\|}$, and $v>0$ is a constant.

Proof: We refer to [17] for a proof.

Remark 1: One should note that dynamical system (8) with continuous vector forces (9) may have some additional unfavorable equilibria. A simple analysis shows that in equilibrium the net force on each particle $p_{i}$ can be zero while some of the force components are not zero (see Fig. 1). In fact, nonzero spring-like forces in equilibrium imply infeasibility of the corresponding solution (path). This verifies the possibility of converging to infeasible solutions (paths). In Section IV, we will show that by employing discontinuous forces such (unfavorable) possibilities can be withdrawn. We will show that all unfavorable equilibria (corresponding to infeasible paths) are unstable.

Remark 2: Some additional restrictions on the initial and final orientations of the vehicle can be imposed. This can be done by fixing the positions of particles $p_{1}$ and $p_{n-1}$ additional to $p_{0}$ and $p_{n}$ by imposing the constraints $m_{2}, m_{n-1}>$ $M$ for some large enough $M>0$.

\section{Stability Analysis of Multi-Particle System WITH DisCONTINUOUS FORCES}

In this section, we use discontinuous elasticity functions and by means of net force analysis in the equilibrium, we show that an equilibrium is stable if and only if all of the forces are equal to zero. We should emphasize that forces are zero if and only if constraints are satisfied. We consider the following class of discontinuous elasticity functions

$$
f(z)=\left\{\begin{array}{ccc}
0 & \text { if } & z \geq \eta \\
-w & \text { if } & z<\eta
\end{array},\right.
$$

where $w, \eta \geq 0$ are constant.

Theorem 2: Consider the multi-particle dynamical system (8) with

$$
\mathbf{F}_{i}=\sum_{\substack{j=0 \\ j \neq i}}^{n} f_{i j}\left(\left\|p_{i}-p_{j}\right\|\right) \mathbf{e}_{i j}-v \dot{p}_{i}
$$

where the elasticity functions $f_{i j}$ are either continuous as in definition 1 or discontinuous as in (10). Then for almost all initial conditions, the trajectories of the multi-particle dynamical system (8) asymptotically converge to an equilibrium. Furthermore, a feasible solution of problem (7) is a locally asymptotically stable equilibrium of the multi-particle dynamical system (8) if all the corresponding spring-like forces are equal to zero.

Proof: We refer to [17] for a proof. 


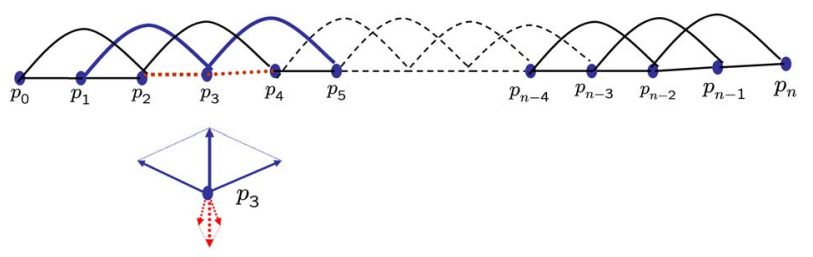

Fig. 1. Analysis of the net forces in the equilibrium which shows that the net forces in $p_{3}$ could be zero even though the forces are not zero.

Theorem 2 shows that for properly chosen continuous elasticity functions the set of all stable equilibria of the multiparticle dynamical system (8) contains all feasible solutions of (7). In the following theorem, it is shown that by means of discontinuous elasticity functions one can actually prove that all unfavorable equilibria of the multi-particle dynamical system (8) are unstable. In other words, theorem (3) shows that for almost all initial conditions (except for those where the particles lie on a straight line passing through $p_{0}$ and $p_{n}$ which constitute a set of measure zero) the trajectories of the multi-particle dynamical system asymptotically converges to an equilibrium which is a feasible solution of problem (7). In the sequel, we consider the following class of discontinuous elasticity functions

$f_{i(i+1)}(z)=\left\{\begin{array}{clc}w_{1} & \text { if } & \left(z-\frac{l}{n}\right) \geq \frac{w_{1}}{k_{f}} \\ k_{f}\left(z-\frac{l}{n}\right) & \text { if } & -\frac{w_{1}}{k_{f}} \leq z \leq \frac{w_{1}}{k_{f}} \\ -w_{1} & \text { if } & \left(z-\frac{l}{n}\right) \leq-\frac{w_{1}}{k_{f}}\end{array}\right.$

and

$$
f_{(i-1)(i+1)}(z)=\left\{\begin{array}{ccc}
0 & \text { if } & z \geq \eta \\
-w_{2} & \text { if } & z<\eta
\end{array}\right.
$$

where $\eta=\frac{l}{n} \sqrt{4-\frac{\kappa_{\max }^{2} l^{2}}{n^{2}}}$ and $w_{1}, w_{2}, k_{f}>0$ are some constant numbers.

Theorem 3: Consider the multi-particle dynamical system described by (8) with $2 k$ particles (i.e., $n=2 k-1$ ) and discontinuous elasticity functions defined as (12) and (13). If $w_{2}>2 w_{1}$, then all of the infeasible equilibria are either unstable or saddle with measure zero region of attraction.

Proof: Without loss of generality, we may assume that $\left\|p_{0}-p_{n}\right\|>l$. If $\left\|p_{0}-p_{n}\right\|<l$, then problem (7) is infeasible. When $\left\|p_{0}-p_{n}\right\|=l$, there is only one stable equilibrium that corresponds to the case where all particles lie on a straight line connecting $p_{0}$ to $p_{n}$.

In Theorem 2, we showed that the multi-particle dynamical system is stable. This means that the trajectories of the multi-particle system converge to stable equilibrium. In an equilibrium point, the net force on a given particle is equal to zero. This does not necessarily means that all elasticity functions acting on that particle are zero. There are two types of springs vector forces: (i) To enforce particles to be equidistant: $f_{i(i+1)}\left(\left\|p_{i}-p_{i+1}\right\|\right) \mathbf{e}_{i(i+1)}$, (ii) To satisfy curvature constraints: $f_{(i-1)(i+1)}\left(\left\|p_{i-1}-p_{i+1}\right\|\right) \mathbf{e}_{(i-1)(i+1)}$. It is easy to see that

$$
\left|f_{i(i+1)}\right| \leq w_{1}<\frac{w_{2}}{2},
$$

and

$$
\left|f_{(i-1)(i+1)}\right|=0 \quad \text { or } \quad w_{2} \text {. }
$$

We can associate a graph to the multi-particle system with nodes representing the mass particles. There is an edge between a pair of nodes if there is a nonzero spring force between the two particles. From Fig. 1, one can see that each particle $p_{i}$ for $i=2, \ldots, 2 k-3$ is connected to four other particles (the positions of the particles $p_{0}$ and $p_{2 k-1}$ are fixed). In an equilibrium point, there are three springlike forces with magnitudes $f_{01}, f_{12}$ and $f_{13}$ associated with node $p_{1}$. Since the net force is zero at this node, we have

$$
f_{01} \mathbf{e}_{01}+f_{12} \mathbf{e}_{12}+f_{13} \mathbf{e}_{13}=\mathbf{0}
$$

It follows that

$$
\left\|f_{01} \mathbf{e}_{01}+f_{12} \mathbf{e}_{12}\right\|=\left|f_{13}\right| .
$$

Assume that $f_{13}=w_{2}$, then from the above equation we get

$$
\left\|f_{01} \mathbf{e}_{01}+f_{12} \mathbf{e}_{12}\right\|=\left|f_{13}\right|=w_{2} .
$$

On the other hand, we have

$$
\left\|f_{01} \mathbf{e}_{01}+f_{12} \mathbf{e}_{12}\right\| \leq\left|f_{01}\right|+\left|f_{12}\right| \leq 2 w_{1}<w_{2} .
$$

This is a contradiction, because from our assumptions we know that $w_{2}>2 w_{1}$. Therefore, we conclude that $f_{13}=$ 0 . In other words, there are only three spring-like forces acting on particle $p_{3}$, i.e., $f_{23}, f_{34}$ and $f_{35}$. Using a similar argument, we can also show that $f_{35}=0$. By repeating the same procedure on the other nodes, it follows that

$$
f_{35}=f_{57}=\ldots=f_{(2 k-3)(2 k-1)}=0 .
$$

Therefore, at nodes with odd indices all spring-like forces resulting from curvature constraints are zero and that can be eliminated from the graph. Similarly, we can argue that at node $p_{2 k-2}$ we have $f_{(2 k-2)(2 k-4)}=0$. By performing a similar analysis, we can show that

$$
f_{(2 k-2)(2 k-4)}=f_{(2 k-4)(2 k-6)}=\ldots=f_{20}=0 .
$$

From (20) and (21), we conclude that all spring-like forces corresponding to curvature constraints are equal to zero. Thus, the only possibility in order to have $f_{i(i+1)} \neq 0$ (for all $i=0, \ldots, 2 k-1)$ in an equilibrium is that all particles to lie on a straight line passing through $p_{0}$ to $p_{2 k-1}$. This formation of particles is clearly saddle because we assumed that $\left\|p_{0}-p_{n}\right\|>l$ and all particles will have expansion forces acting on them and infinitesimal deviation from the line push the particles further away from line which makes the formation unstable.

Remark 3: In the proof of Theorem 3, we assumed that net force is equal to zero in an equilibrium. In continuous systems, this is always the case. However, the vector field could be nonzero in an equilibrium of a nonsmooth dynamical system. We should note that we proved in Theorem 2 that for almost all initial conditions the trajectory of the multiparticle dynamical system converges to a stable equilibrium. Therefore, we only need to show that if the net force is not 


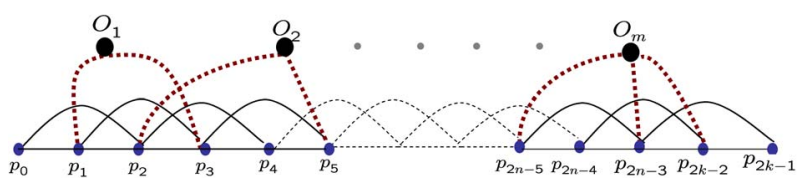

Fig. 2. Graph representation of the forces in the presence of obstacles.

zero in an equilibrium, then the equilibrium is either unstable or is saddle with measure zero region of attraction. We refer to [17] for a formal proof and further discussion on this issue. We should note that a similar result holds for the equilibria of the the multi-particle dynamical system defined in Theorems 4 and 5.

\section{Single-Vehicle Path Planning in Presence of MOVING OBSTACLES}

In this section, our goal is to find a path for Dubins' vehicle in presence of moving obstacles with known motion trajectories. We assume that each obstacle $j$ can be represented by a disk $\mathbf{O}_{\mathbf{j}}\left(c_{j}(t), r_{j}(t)\right)$ for all $j=1, \ldots, M$. Furthermore, we assume that at any time instant these disks are not overlapping. Suppose that $t_{i}$ is the time instant at which the vehicle is at waypoint $p_{i}$. Therefore, the obstacle avoidance condition can be written as follow:

$$
\left\|p_{i}-c_{j}\left(t_{i}\right)\right\| \geq r_{j}\left(t_{i}\right),
$$

for all $i=0, \ldots, n$ and $j=1, \ldots, M$. Similar to the static obstacles, in order to enforce obstacle avoidance constraints, we define a new spring-like force between obstacle $\mathbf{O}_{j}\left(c_{j}(t), r_{j}(t)\right)$ and particle $p_{i}$ as $f_{i j}\left(\left\|p_{i}-c_{j}\left(t_{i}\right)\right\|\right) \mathbf{e}_{i j}$ with the following elasticity function

$$
f_{i j}(z)=\left\{\begin{array}{rr}
0 & \text { if } \quad z \geq r_{j}\left(t_{i}\right) \\
-w_{3} & \text { otherwise }
\end{array} .\right.
$$

Similar to the static obstacle case, the elasticity functions defined by (23) belong to the class of elasticity functions defined by (10). Therefore, the stability conditions of Theorem (2) hold and the trajectories of the multi-particle dynamical system (8) with new obstacle-avoidance forces asymptotically converge to equilibrium.

Theorem 4: Consider the multi-particle dynamical system (8) with $2 k$ particles and $M$ moving obstacles with known motion trajectories. The obstacles are represented by nonoverlapping disks $\mathbf{O}_{\mathbf{j}}\left(c_{j}(t), r_{j}(t)\right)$ for $j=1, \ldots, M$. If the spring-like forces and obstacle avoidance forces are defined as (12), (13), and (23) with the following constraints

$$
\begin{aligned}
2 w_{1} & <w_{2}, \\
2\left(w_{1}+w_{2}\right) & <w_{3},
\end{aligned}
$$

then all stable equilibria of the multi-particle dynamical system (8) are feasible.

Proof: We only need to show that in an equilibrium all the particles lie outside the obstacles. Then from Theorem 3 it follows that all stable equilibria are feasible. For this purpose, we assume that particle $p_{i}$ is inside the obstacle with center $c_{j}\left(t_{i}\right)$. This means that there are five force components acting on particle $p_{i}$ with zero net force,

$$
\begin{aligned}
& \underbrace{f_{(i-1) i} \mathbf{e}_{(i-1) i}+f_{i(i+1)} \mathbf{e}_{i(i+1)}}_{\text {spring forces to enforce particles to be equidistant }} \\
& +\underbrace{f_{(i-2) i} \mathbf{e}_{(i-2) i}+f_{i(i+2)} \mathbf{e}_{i(i+2)}}_{\text {spring forces to impose curvature constraints }} \\
& -\underbrace{w_{3} \mathbf{e}_{i j}}_{\text {obstacle avoidance force }}=\mathbf{0}
\end{aligned}
$$

It follows that

$$
\begin{aligned}
& \| f_{(i-1) i} \mathbf{e}_{(i-1) i}+f_{i(i+1)} \mathbf{e}_{i(i+1)} \\
& +f_{(i-2) i} \mathbf{e}_{(i-2) i}+f_{i(i+2)} \mathbf{e}_{i(i+2)}\|=\| w_{3} \mathbf{e}_{i j} \|=w_{3} .
\end{aligned}
$$

On the other hand,

$$
\begin{aligned}
& \| f_{(i-1) i} \mathbf{e}_{(i-1) i}+f_{i(i+1)} \mathbf{e}_{i(i+1)} \\
&+f_{(i-2) i} \mathbf{e}_{(i-2) i}+f_{i(i+2)} \mathbf{e}_{i(i+2)} \| \\
& \leq\left\|f_{(i-1) i} \mathbf{e}_{(i-1) i}\right\|+\left\|f_{i(i+1)} \mathbf{e}_{i(i+1)}\right\|+ \\
&\left\|f_{(i-2) i} \mathbf{e}_{(i-2) i}\right\|+\left\|f_{i(i+2)} \mathbf{e}_{i(i+2)}\right\| \\
& \leq w_{1}+w_{1}+w_{2}+w_{2}=2\left(w_{1}+w_{2}\right)<w_{3}
\end{aligned}
$$

Thus, it follows that $w_{3}<w_{3}$ which is a contradiction. Therefore, in an equilibrium there are no force components between the particles and the obstacles. Therefore, all the particles lie outside the obstacles.

\section{Vi. Multi-Vehicle Path Planning in Presence of Moving ObStaCles}

The developed framework in previous sections can be employed to handle multi-vehicle path planning problem in presence of moving obstacles. We associate a multi-particle dynamical system (representing a path) with each vehicle. For example, for $N$ vehicles we need to have $N$ different multi-particle dynamical systems. In order to guarantee a collision-free path for each vehicle, we need to introduce a new force component so called collision-avoidance force.

Consider two vehicles with constant speeds $V_{1}$ and $V_{2}$ traveling distances $l_{1}$ and $l_{2}$. The corresponding waypoints for these two vehicles are represented by $\left\{p_{0}^{1}, p_{1}^{1}, \ldots, p_{n}^{1}\right\}$ and $\left\{p_{0}^{2}, p_{1}^{2}, \ldots, p_{m}^{2}\right\}$. In order to avoid collision between the two vehicles, we must guarantee that both vehicles are not going to arrive at a waypoint simultaneously. In other words, if there exist waypoints $p_{i}^{1}$ and $p_{j}^{2}$ for which

$$
\left|\frac{l_{1}}{n V_{1}} i-\frac{l_{2}}{m V_{2}} j\right| \leq \epsilon
$$

for some time-error $\epsilon>0$, then the following constraint has to be imposed on the corresponding waypoints

$$
\left\|p_{i}^{1}-p_{j}^{2}\right\| \geq \epsilon^{\prime}
$$

for some position-error $\epsilon^{\prime}>0$. Therefore, we can introduce new elasticity functions for all pairs of points $p_{i}^{1}$ and $p_{j}^{2}$ satisfying condition (25) as follows

$$
f_{i j}(z)=\left\{\begin{array}{cc}
0 & \text { if } \\
-w_{4} & \text { otherwise }
\end{array}\right.
$$


We emphasize that the interaction force (27) is defined between waypoints of two different trajectories. According to Theorem 2, it is straightforward to show that the resulting multi-particle dynamical systems under collision-avoidance constraints (forces) are stable. Furthermore, the following theorem shows that all unfavorable equilibria of the overall system are unstable.

Theorem 5: Suppose that $N$ is the number of vehicles, $M$ the number of moving obstacles, and $2 k_{i}$ the number of particles representing a trajectory for vehicle $i$ for $i=1, \ldots, N$. Each obstacle is represented by a disk $\mathbf{D}\left(c_{j}(t), r_{j}(t)\right)$ for $j=1, \ldots, M$. At any given time, assume that no more than two vehicles can possibly collide. Then all stable equilibria of the resulting multi-particle dynamical system with elasticity functions defined by (12), (13), (23) and (27) in which

$$
\begin{aligned}
2 w_{1} & <w_{2} \\
2\left(w_{1}+w_{2}\right) & <w_{3} \\
2\left(w_{1}+w_{2}\right)+w_{3} & <w_{4}
\end{aligned}
$$

are feasible.

Proof: We only need to prove that in an equilibrium paths are collision-free. Then according to Theorem 4 one can conclude that all stable equilibria are feasible. Let assume that vehicles 1 and 2 collide, i.e., there exist waypoints $p_{i}^{1}$ and $p_{j}^{2}$ that satisfy (25) and $\left\|p_{i}^{1}-p_{j}^{2}\right\| \leq \epsilon^{\prime}$. In this case, there are (at most) six force components acting on particle $p_{i}^{1}$ with zero net force (in the following equations for the sake of simplicity superscript 1 is dropped)

$$
\begin{aligned}
& \underbrace{f_{(i-1) i} \mathbf{e}_{(i-1) i}+f_{i(i+1)} \mathbf{e}_{i(i+1)}}_{\text {spring forces to enforce particles to be equidistant }} \\
& +\underbrace{f_{i(i+2)} \mathbf{e}_{i(i+2)}+f_{(i-2) i} \mathbf{e}_{(i-2) i}}_{\text {spring forces to impose curvature constraints }} \\
& \quad \underbrace{f_{i k} \mathbf{e}_{i k}}_{-}
\end{aligned}
$$

for some obstacle with index $k$. It follows that

$$
\begin{aligned}
\| f_{(i-1) i} \mathbf{e}_{(i-1) i} & +f_{i(i+1)} \mathbf{e}_{i(i+1)}+f_{i(i+2)} \mathbf{e}_{i(i+2)} \\
& +f_{(i-2) i} \mathbf{e}_{(i-2) i}-f_{i k} \mathbf{e}_{i k}\|=\| w_{4} \mathbf{e}_{i j} \|=w_{4}
\end{aligned}
$$

Therefore, we have

$$
\begin{gathered}
\left\|w_{4} \mathbf{e}_{i j}\right\| \leq \quad\left\|f_{(i-1) i} \mathbf{e}_{(i-1) i}\right\|+\left\|f_{i(i+1)} \mathbf{e}_{i(i+1)}\right\|+ \\
\left\|f_{i(i+2)} \mathbf{e}_{i(i+2)}\right\|+\left\|f_{(i-2) i} \mathbf{e}_{(i-2) i}\right\|+\left\|f_{i k} \mathbf{e}_{i k}\right\| \\
\leq w_{1}+w_{1}+w_{2}+w_{2}+w_{3}=2\left(w_{1}+w_{2}\right)+w_{3}<w_{4}
\end{gathered}
$$

This is a contradiction. Therefore, the collision-avoidance force $f_{i j}$ must be equal to zero. This means that in equilibrium all paths are collision free.

\section{CONCLUSION}

We formulated an arbitrarily fine relaxation of the path planning problem for nonholonomic vehicles as a nonconvex feasibility optimization problem. Then, we proposed a nonsmooth dynamical systems approach to find feasible solutions of the nonconvex optimization problem. We showed that the set of equilibria of the nonsmooth dynamical systems contains all feasible solutions of the optimization problem and that the dynamical system is asymptotically stable. This method can be applied to compute feasible paths for multi vehicles in presence of moving obstacles.

\section{REFERENCES}

[1] J. C. Latombe. Robot Motion Planning. Kluwer Academic Publishers, 1991

[2] S. M. LaValle. Planning Algorithms. Cambridge University Press, 2006

[3] L. E. Dubins, On curves of minimal length with a constraint on average curvature, and with prescribed initial and terminal positions and tangents, Amer. J. Math., vol. 79, no. 3, pp. 497-516, 1957

[4] J. Reif and H. Wang. The complexity of the two dimensional curvatureconstrained shortestpath problem. In WAFR 98: Proceedings of the third workshop on the algorithmic foundations of robotics on Robotics, Natick, MA, USA, 1998

[5] S. Fortune and G. Wilfong. Planning constrained motion. Ann. Math. Artificial Intelligence, 3(1):2182, 1991

[6] P. Jacobs and J. Canny. Planning smooth paths for mobile robots. In Proceedings of the IEEE International Conference on Robotics and Automation, 1989.

[7] H. Wang and P. K. Agarwal. Approximation algorithms for curvatureconstrained shortest paths. In Proceedings of the seventh annual ACMSIAM symposium on Discrete algorithms, Philadelphia, PA, USA, 1996.

[8] D. E. Koditschek and E. Rimon, Robot navigation functions on manifolds with boundary, Advances Appl. Math., vol. 11, 1990.

[9] S. Sastry, Nonlinear Systems , Springer, 2004.

[10] A. F. Filipov, Differential equations with discontinuous right-hand side, Amer. Math. Soc. Translations, vol. 42, no. 2, pp. 191-231, 1964.

[11] F.H. Clarke, Yu.S. Ledyaev, R.J. Stern, and P.R. Wolenski. Nonsmooth Analysis and Control Theory.Graduate Texts in Mathematics; 178. Springer, New York, 1998.

[12] A. F. Filippov. Differential equations with discontinuous right-hand side. Mathematics and Its Applications (Soviet Series). Kluwer Academic Publishers, The Netherlands, 1988.

[13] J. Cortes, Discontinuous dynamical systems - a tutorial on solutions, nonsmooth analysis, and stability IEEE Control Systems Magazine, 2008 , to appear.

[14] D. Shevitz and B. Paden. Lyapunov stability theory of nonsmooth systems. IEEE Transactions on Automatic Control, 39(9):19101914,1994.

[15] I. Gohberg, P. Lancaster and L Rodman. Invariant Subspaces of Matrices with Applications, SIAM Classics in Applied Mathematics, 2006.

[16] F. H. Clarke, Optimization and Nonsmooth Analysis. Canadian Mathematical Society Series of Monographs and Advanced Texts, John Wiley, 1983.

[17] A. Ahmadzadeh, N. Motee, A. Jadbabaie, and G. Pappas. Elastic Multi-Particle Systems for Multi-Vehicle Path Planning - Part I: Theory. IEEE Trans. on Automatic Control, Submitted, 2009. 